\title{
Analytical Ultracentrifugation of Model Nanoparticles: Comparison of Different Analysis Methods ${ }^{\mathrm{a}}$
}

\author{
Vikas Mittal, Antje Völkel, ${ }^{*}$ Helmut Cölfen
}

Sedimentation analysis of nanoparticle $\left(\mathrm{ZrO}_{2}\right.$ and $\left.\mathrm{SiO}_{2}\right)$ suspensions of different particle sizes in various solvents as nanoparticle model systems was carried out using interference optics in the analytical ultracentrifuge. The particles differed in their morphology: $\mathrm{SiO}_{2}$ particles were spherical, whereas $\mathrm{ZrO}_{2}$ particles were in one case spherical and in the second case non-spherical and spherical. Different analysis programs, based on different principles of data analysis, were used for the evaluation of the size distributions of these particles, viz. SEDFIT $\left[l s-g^{*}(s), c(s)\right]$, UltraScan (vHW, 2DSA-MC), SedAnal (dcdt) and VelXLAI (GFL) method and SedAnal, in order to ascertain the benefits and limitations of these analysis methods in characterising the examined nanoparticles.

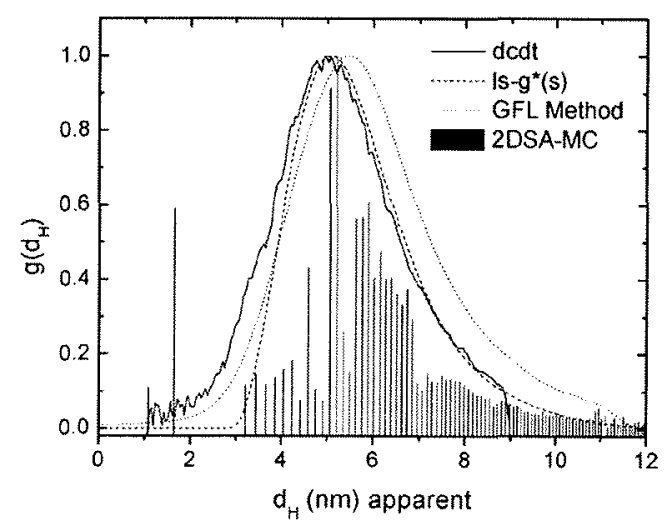

\section{Introduction}

The size and its distribution for organic and inorganic particles are required to be accurately known as these parameters drive their use in multiple applications. A number of characterization techniques have been developed for the determination of the particle size and particle size distribution which include analytical ultracentrifugation, field flow fractionation, dynamic light scattering, etc.

A. Völkel, H. Cölfen

Max-Planck Institute of Colloids and Interfaces, Colloid Chemistry, 14476 Golm, Germany

Fax: (+49) 3315679502; E-mail: antje.voelkel@mpikg.mpg.de V. Mittal

BASF SE, Polymer Research, 67056 Ludwigshafen, Germany
Analytical ultracentrifugation is a preferred technique for many systems owing to high statistical capability and versatility to analyze a wide spectrum of particle systems. ${ }^{[1-4]}$ A sedimentation velocity experiment is the commonly used technique for the determination of the sedimentation profile as well as size distribution of the sample. By using different detection optics e.g. absorption or interference, ${ }^{[5-8]}$ the sedimentation of the particles or dissolved macromolecules according to their size or density can be monitored owing to the generated radial concentration gradient as a function of time. ${ }^{[9-13]}$ The experiment allows the generation of a step like concentration profile in the ultracentrifuge cell corresponding to one species. Depending on the speed, the sedimentation boundary is broadened by diffusion. The sedimentation coefficient $s$ is classically defined as

$$
\begin{gathered}
s=u / \omega^{2} r \\
\text { or } \\
s=\left(\mathrm{d} r_{\mathrm{bnd}} / \mathrm{d} t\right) / \omega^{2} r=\ln \left(r_{\mathrm{bnd}} / r_{\mathrm{m}}\right) /\left(\omega^{2} t\right)
\end{gathered}
$$


where $r$ is the radial distance from the centre of rotation, index $m$ the meniscus, $\omega$ the angular velocity and $d r_{b n d} / d t$ represents the velocity of the boundary.

The plot of $\left(\ln r_{\mathrm{bnd}} / r_{\mathrm{m}}\right)$ versus time, $t$ is a straight line, from the slope of which the value of the sedimentation coefficient can be calculated. However, the samples in real life situations have multiple components and broad distributions of size or molecular weight. In such cases, the use of the above mentioned weight average sedimentation coefficient, which better describes monomodal systems, is not sufficient to characterize the sample and it is important to determine the whole sedimentation coefficient distribution.

Various methods have been developed to analyse sedimentation velocity data to generate sedimentation coefficient distributions and the respective particle size distributions for the colloidal particles. Also, when the particles are generally smaller than $30 \mathrm{~nm}$, diffusion broadening of the sedimenting boundaries occurs due to the high diffusion coefficient of the small particles. Partly, analysis principles also perform the separation of the diffusion effects from the sedimentation velocity data i.e. to remove the boundary spreading by diffusion from the spreading by a distribution in particle size. In the following, these principles for the sedimentation velocity analysis are described:

\section{Lamm Equation Fitting}

This principle is based on a boundary model for sedimentation and subsequently size or molecular mass analysis by generating numerical solutions to the Lamm Equation which governs both sedimentation and diffusion.

$$
\frac{\partial c}{\partial t}=D\left(\frac{\partial^{2} c}{\partial r^{2}}+\frac{1}{r} \frac{\partial c}{\partial r}\right)-\omega^{2} s \underset{\text { diffusionterm }}{\left(r \frac{\partial c}{\partial r}+2 c\right)}
$$

Initial values of sedimentation coefficient, frictional coefficient and the partial specific volume for the solute are guessed and are used to calculate finite element solutions for a large number of species with varying $s$ values. ${ }^{[3,14-17]}$ These calculated data are then adjusted to experimental data by maximum entropy regularisation to generate a sedimentation coefficient distribution $c(s)$ and is implemented in SEDFI ${ }^{[18-20]}$ [continuous $c(s)$ distribution]. This analysis also allows the investigation of polydisperse particles as well as multi-component systems. ${ }^{[18-20]}$

Also the two dimensional spectrum analysis for sedimentation velocity experiments is based on the Lamm equation. With a model free grid search of $s$ and $f / f_{0}$ and a following Monte Carlo approach [UltraScan (2DSA-MC)] ${ }^{[21]}$ to calculate the confidence limit of each solute or regularisation $\left\{\text { SEDFrT }\left[c\left(s, f f_{0}\right)\right]\right\}^{[22]}$ it is implemented in the software packages.

\section{van Holde-Weischet Function}

Another widely used principle for the generation of sedimentation coefficient distributions is the van Holde-Weischet function. ${ }^{[23]}$ It is based on the extrapolation to infinite time to separate the effect of diffusion from sedimentation because sedimentation is proportional to time, whereas the diffusion is proportional to the square root of time. The following Equation provides the basis of the analysis:

$$
s_{w}^{*}=s-\frac{D^{1 / 2}}{r_{0}} \frac{2}{\omega^{2}} \phi^{-1}(1-2 w) t^{-1 / 2}
$$

where $\Phi$ is the error function, $s_{w}^{*}$ the apparent weight average sedimentation coefficient and $r_{0}$ is the meniscus position. A number of data points from one sedimentation velocity scan at a defined time are selected such that they are evenly placed between the baseline and the plateau where $w$ is the relation between the plateau concentration and the concentration at a radial distance $r_{w} . s_{w}^{*}$ is then calculated for each of the data points. These values are then plotted as a function of inverse root of run time which generates a typical van Holde-Weischet plot. ${ }^{[23]}$ Linear extrapolation of the corresponding $s_{w}^{*}$ values for the same position between the baseline and plateau at different times to infinite time is then performed. The intersection with the $y$-axis yields the diffusion corrected sedimentation coefficient for the particular position between the baseline and the plateau from where the data points were selected. By combining these values, a complete diffusion corrected sedimentation coefficient distribution can be determined. Thus, this principle is a model free transformation of the sedimentation velocity data into a sedimentation coefficient distribution from which the effect of diffusion broadening of the sedimentation boundary has been separated. This method (vHW) gives a sedimentation coefficient distribution $g(s)$ and is implemented in the SEDFrT and the UltraScan software. ${ }^{[20,24]}$ It is also an efficient test if homogeneity or non-ideality are present in the samples. For a single monodisperse component in the samples, one value of sedimentation coefficient is obtained because of the intersection of the lines on the $y$-axis at one point. For multiple components and polydispersity present in the sample, a number of intersection points corresponding to a position between the baseline and plateau are achieved, integration of which yields the complete sedimentation coefficient distribution of the sample components. ${ }^{[21-26]}$ 


\section{Gosting Fujita Lechner (GFL) Principle}

In this principle, the sedimentation coefficient distribution $g(s, c, t)$ can be determined according to:

$$
g(s, c, t)=\left(1 / c_{0}\right)[\mathrm{d} c(r, t) / \mathrm{d} r]\left(r / r_{m}\right)^{2} r \int \omega^{2} \mathrm{~d} t
$$

where $c_{0}$ is the initial (loading) concentration of the particles or polymers and $c(r, t)$ the concentration at distance $r$ and running time $t^{[27-30]}$

The calculation of the sedimentation coefficient distribution $g(s, c)$ from $g(s, c, t)$ considers that the sedimentation of particles or macromolecules in dispersion or solution is proportional to the running time $t$ and the diffusion proportional to the square root of the time $t^{1 / 2}$. That means extrapolation against $t \rightarrow \infty$ or $1 / t \rightarrow 0$ corresponds to pure sedimentation and extrapolation against $t \rightarrow 0$ to pure diffusion. The extrapolation of the sedimentation coefficient distribution $g(s, c, t)$ or its integral function $G(s, c, t)$ to $1 /$ $t \rightarrow 0$ gives directly and model free the distribution functions $g(s, c)$ and $G(s, c)$.

The recovery rate $c / c_{0}$ (measured concentration/initial concentration) can be determined by linear extrapolation of the fringe shift $J$ as a function of time $t$ at the local evaluation end $R_{\mathrm{e}}$. The GFL principle is implemented in the software package VelXLAI.

\section{Time Derivative Principle}

A significantly improved signal to noise ratio of the experimental data can be achieved in the time derivative function. ${ }^{[31-33]}$ This is a result of the subtraction of the neighbouring scans from each other which helps to reduce the optical errors and random noise. The time derivative function provides the differential form $g^{*}(s)$ of the sedimentation coefficient distribution by determining the time derivative of the neighbouring radial scans acquired at different times according to the following equation:

$$
g^{*}(s)_{t}=\left(\frac{\partial\left\{c(r, t) / c_{0}\right\}}{\partial t}\right)\left(\frac{\omega^{2} t^{2}}{\ln \left(r_{m} / r\right)}\right)\left(\frac{r}{r_{m}}\right)^{2}=\frac{\partial G^{*}(s)_{t}}{\partial s}
$$

which is a combination of the following Equation (6) and (7):

$$
\begin{aligned}
& \ln \left(r_{\text {bnd }} / r_{m}\right)=s \omega^{2} \mathrm{t}=s \int_{0}^{t} \omega^{2} \mathrm{~d} t \\
& g(s)=\frac{d\left(c(s) / c_{0}\right)}{\mathrm{d} s}\left(\frac{r}{r_{m}}\right)^{2}=\frac{\partial G(s)}{\partial s}
\end{aligned}
$$

where $g^{*}(s)$ is the apparent differential distribution. It is implemented in the software packages SEDFIT as least square direct boundary modelling $\left[l s-g^{*}(s)\right]$ and SedAnal as well as UitraScan (dcdt). It equals the true distribution if no significant diffusion is observed. In the cases, where the effects of diffusion cannot be ignored, the extrapolation of $g\left(s^{*}, t\right)$ curves to infinite time yields the true distribution $g(s)$. This principle can be used accurately for diluted samples, interacting systems as well as polydisperse systems where the sedimentation coefficients span over orders of magnitude.

Every method has, based on different algorithms and noise handling, its own strengths and limitations. Therefore, the objective of the current study is to compare the performance of the above mentioned sedimentation velocity data analysis methods and thus programs for inorganic model particles of various particle sizes and size distributions from the user's point of view. The inorganic systems have been chosen as models for solid nanoparticles because they were not only available with different size and size distribution but also with different shapes.

\section{Experimental Part}

Two kinds of model zirconium dioxide $\left(\mathrm{ZrO}_{2}\right)$ particles were kindly supplied by Prof. Georg Garnweitner, Partikeltechnik, TU Braunschweig, Germany. ${ }^{[34,35]}$ The particles were designated as $\mathrm{ZrO}_{2}-1$ and $\mathrm{ZrO}_{2}-2$, respectively. $\mathrm{ZrO}_{2}-1$ had a suspension medium of tetrahydrofuran whereas particles in $\mathrm{ZrO}_{2}-2$ sample were suspended in ethanol. Similarly, two different kinds of silica particles, named as silicon dioxide $\left(\mathrm{SiO}_{2}-1\right.$ and $\left.\mathrm{SiO}_{2}-2\right)$, in water were procured by BASF $\mathrm{SE}$, Germany. From the available materials data on these particles, these differed significantly from each other in particle size and its distribution. An OPTIMA XL-I from Beckman Coulter fitted with interference detection optics (temperature $25^{\circ} \mathrm{C}$, wavelength $675 \mathrm{~nm}$ ) was used for the sedimentation velocity experiments on the particles. Rotational speeds of 30000 rotations per minute and a scan interval of $10 \mathrm{~s}$ were used for the sedimentation experiments with $\mathrm{ZrO}_{2}-1, \mathrm{ZrO}_{2}-2$ and $\mathrm{SiO}_{2}-1$ particles. A speed of 10000 rotations per minute and a scan time interval of $10 \mathrm{~s}$ were used for the $\mathrm{SiO}_{2}-2$ particles owing to their larger particle size. The optimal speed for the experiments has been chosen by gradual acceleration and simultaneous observation of the starting sedimentation at the online monitor of the interference optics to a speed where an estimated scan amount between 50 and 100 scans could be recorded while the particles are sedimenting. The $\mathrm{SiO}_{2}$ samples were measured at a concentration of $10 \mathrm{~g} \cdot \mathrm{L}^{-1}$, whereas $0.5 \mathrm{~g} \cdot \mathrm{L}^{-1}$ concentration was used for the zirconium dioxide particles. Apart from that, $\mathrm{ZrO}_{2}-1$ particles were also measured at additional concentrations of 2.5 and $5 \mathrm{~g} \cdot \mathrm{L}^{-1}$. Distilled water was used for the dilution of the $\mathrm{SiO}_{2}$ particles to achieve the required concentration. The sedimentation velocity data were analysed by using different analysis programs SEDFIT $\left[l s-g^{*}(s), c(s)\right]$, UltraScan (vHW, 2DSA-MC), SedAnal (dcdt) and VelXI.AI (GFL). A density value of $5.89 \mathrm{~g} \cdot \mathrm{cm}^{-3}$ for baddeleyite was used for the zirconium dioxide particles and $\mathrm{d} n / \mathrm{dc}$ of 0.1744 and $0.1849 \mathrm{~cm}^{3} \cdot \mathrm{g}^{-1}$, respectively for $\mathrm{ZrO}_{2}-1$ and $\mathrm{ZrO}_{2}-2$ particles. ${ }^{[36]} \mathrm{A}$ density value of $2.53 \mathrm{~g} \cdot \mathrm{cm}^{-3}$ 
and $\mathrm{d} n / \mathrm{d} c$ value of $0.067 \mathrm{~cm}^{3} \cdot \mathrm{g}^{-1}$ were used for the $\mathrm{SiO}_{2}$ particles. $\mathrm{The} \mathrm{d} n / \mathrm{d} c$ for all samples were measured with a refractometer RFM 340 (Bellingham Stanley Limited) and the density values for the $\mathrm{SiO}_{2}$ particles were measured in a densitometer DMA $5000 \mathrm{M}$ (Anton Paar).Various internal parameters of each analysis program were chosen in order to achieve a good fit of the experimental data.

\section{Results and Discussion}

To study the size distributions of particles using different data analysis methods, $\mathrm{ZrO}_{2}$ and $\mathrm{SiO}_{2}$ particles were used as model systems for solid nanoparticles of biological or synthetic origin. Apart from the differences in the chemistry of the particles or the suspension media in which they are dispersed, the particles also had different shapes. The $\mathrm{ZrO}_{2}-2$ particles were non-spherical and spherical in nature, whereas $\mathrm{ZrO}_{2}-1$ particles were spherical. $\mathrm{SiO}_{2}$ particles were spherical and there were no differences in the surface morphology of the two types of $\mathrm{SiO}_{2}$ particles. The microscopic investigation of the particles carried out by transmission electron microscopy is demonstrated in Figure 1. It should also be noted that the analysis a)

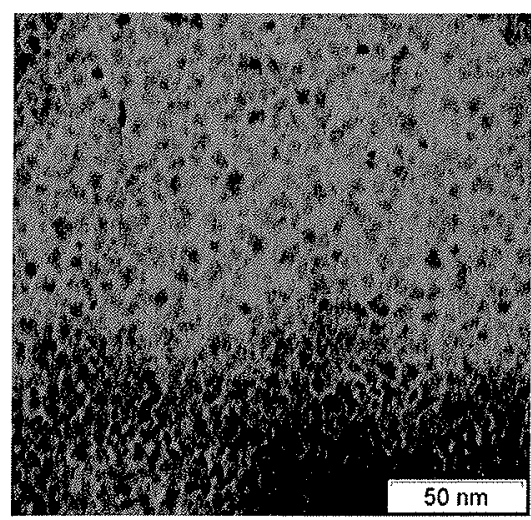

c)

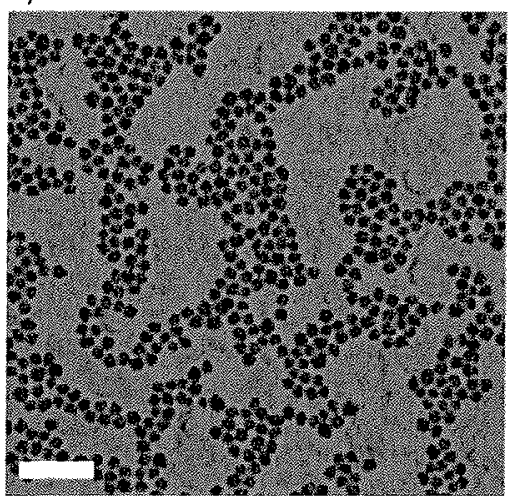

b)

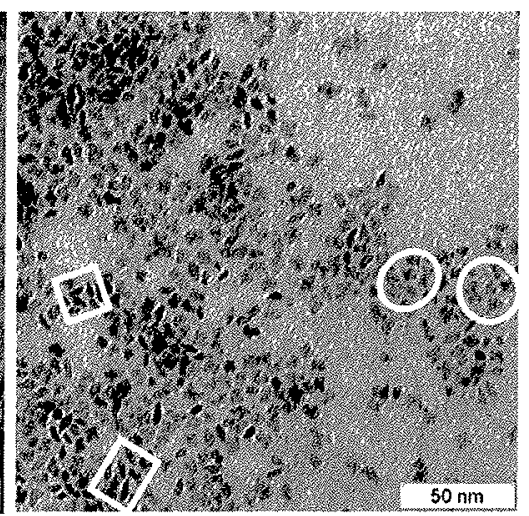

d)

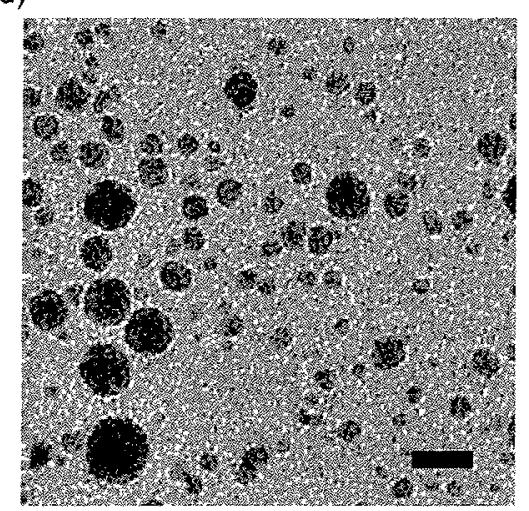

Figure 1. TEM micrographs of a) $\mathrm{ZrO}_{2}-1$, b) $\mathrm{ZrO}_{2}-2$, c) $\mathrm{SiO}_{2}-1$ (the bar reads $35 \mathrm{~nm}$ ) and d) $\mathrm{SiO}_{2}-2$ (the bar reads $100 \mathrm{~nm}$ ) particles. of only one component (monomodal samples) represents the simplest scenario for the comparison of the different analysis methods. However, such an analysis is required to understand the subtleties and relative comparisons of different analysis methods well enough to be able to use them further for more complex mixtures and very polydisperse samples.

Comparisons of the sedimentation velocity profiles for $\mathrm{ZrO}_{2}-1$ and $\mathrm{SiO}_{2}-2$ samples as a function of cell radius and sedimentation time revealed that the later scans in the case of $\mathrm{SiO}_{2}$ particles were broader or more shallow in nature (Figure SI-I of Supporting Information). A clear sedimentation boundary with a lower and upper plateau within one scan was not formed, which indicates that the sample exhibits polydispersity. The scans in case of the zirconium dioxide sample on the other hand formed a more uniform boundary indicating that the particles had a lower polydispersity than the $\mathrm{SiO}_{2}-2$ counterpart. It should also be noted that the $\mathrm{ZrO}_{2}-1$ and $\mathrm{SiO}_{2}-2$ particles were measured at different concentration levels of 0.5 and $10 \mathrm{~g} \cdot \mathrm{L}^{-1}$, respectively due to the largely different refractive index increments. Apart from that, $\mathrm{ZrO}_{2}-1$ particles were also measured at additional concentrations of 2.5 and $5 \mathrm{~g} \cdot \mathrm{L}^{-1}$ in order to ascertain the concentration dependence of the particles on the particle size distributions calculated by different analysis methods (see Supporting Information).

Sedimentation coefficient distributions further provide useful informations on the nature of the particles and the informations are also more quantitative. Figure 2 depicts the sedimentation coefficient distributions of the samples $\mathrm{ZrO}_{2}-$ 1 and $\mathrm{SiO}_{2}-2$ (from SEDFrT). The particles sediment owing to their size, shape as well as density, thus, the sedimentation coefficient distribution is also an indication of the polydispersity in the size of the particles (assuming the density and the shape of the particles to be fairly constant). The $\mathrm{ZrO}_{2}-1$ particles exhibit a narrow sedimentation coefficient distribution in the range of $10-800 \mathrm{~S}$ as compared to the broad distribution in the case of $\mathrm{SiO}_{2}-2$ particles in the range of 200-10000 Sindicating that the particles in $\mathrm{SiO}_{2}-2$ samples are more polydisperse in size as compared to the $\mathrm{ZrO}_{2}-1$ particles. This also confirms the observations from the raw sedimentation velocity data.

Figure $3 a$ shows the particle size distribution of the $\mathrm{ZrO}_{2}-1$ particles resulting 


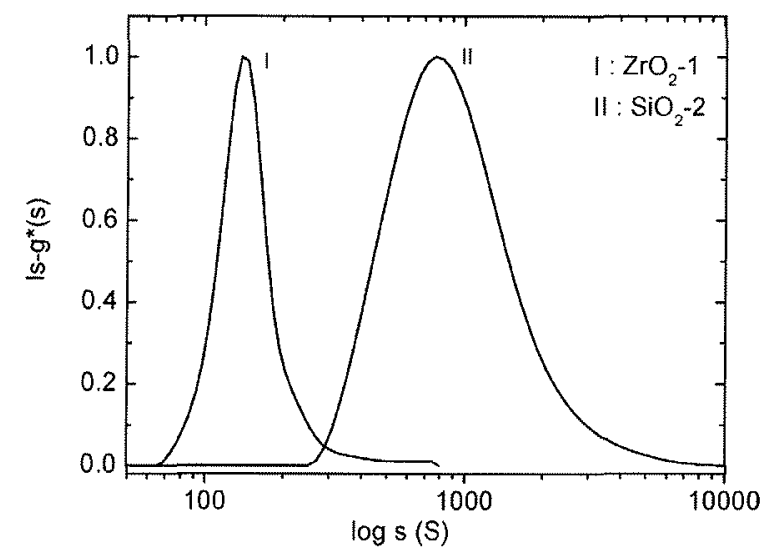

Figure 2. Sedimentation coefficient profiles of $\mathrm{ZrO}_{2}-1$ and $\mathrm{SiO}_{2}-2$ representing the $s$-value range of the particles on a logarithmic scale $\left[I s-g^{*}(s)\right.$ SEDFIT].

a)
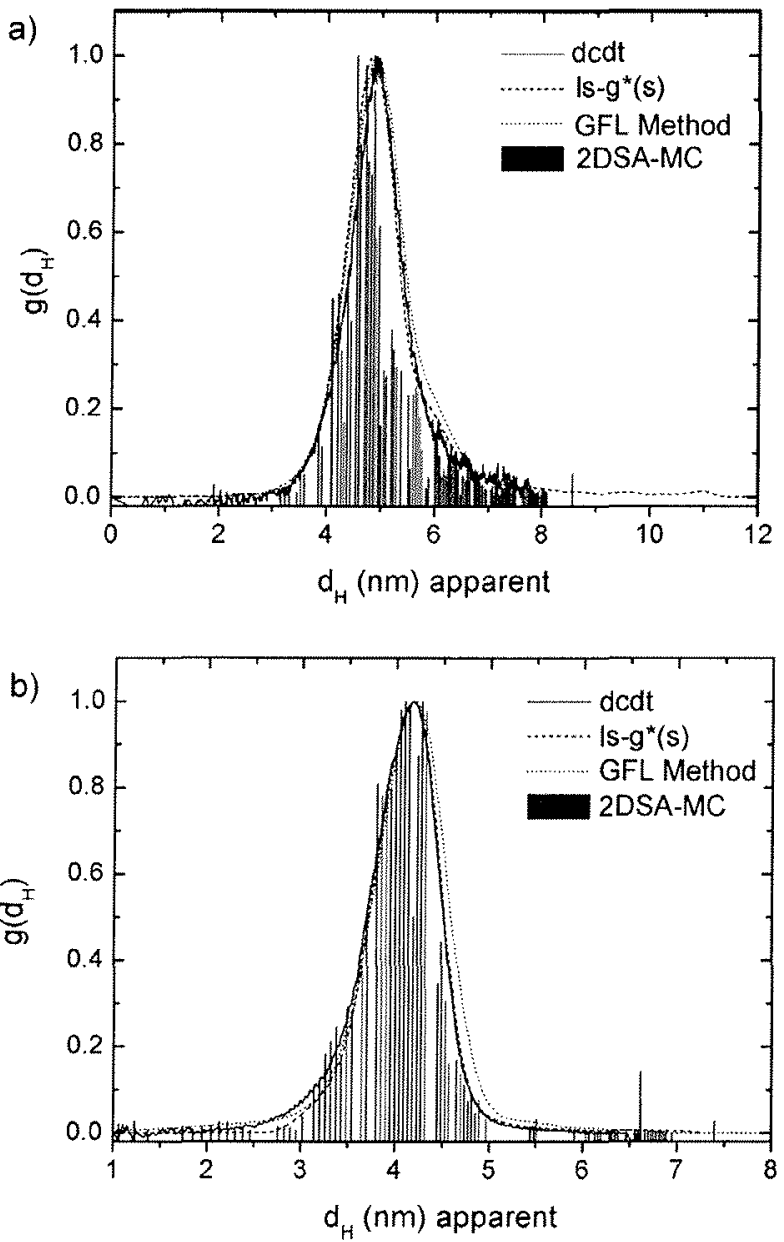

Figure 3. Particle size distributions of a) $\mathrm{ZrO}_{2}-1$ and b) $\mathrm{ZrO}_{2}-2$ particles using different sedimentation velocity data analysis methods. from different analysis methods. As mentioned earlier the $\mathrm{ZrO}_{2}-1$ particles are spherical in nature. Therefore, the analysis of these particles as spheres in the different analysis programs correlates well with the particle morphology. The different analysis programs with their implemented methods SEDFIT $\left[l s-g^{*}(s)\right]$, UltraScan (2DSA-MC), SedAnal (dcdt) and VelXLAI (GFL) are observed to yield similar particle size distributions in the sample. However, the distribution curve in case of 2DSA-MC analysis was observed to be shifted slightly to lower diameters $(\sim 4.7 \mathrm{~nm})$ as compared to other methods which demonstrate a value of $4.8 \mathrm{~nm}$ for the particles at the peak maximum. It has to be noted, that the grid like evaluation of the scans with following Monte Carlo approach does not give a continuous distribution but a step like one with the frequency of each single point depending on the resolution or grid repetition. The particle size range exhibited by $\left[l s-g^{*}(s)\right]$ also runs to higher diameter values as compared to other analysis methods, however the fraction of particles in this size range is insignificant, thus, indicating that for monomodal and fairly monodisperse inorganic particles, the different analysis methods resulted in similar particle size distributions.

Figure $3 \mathrm{~b}$ in analogy shows the particle size distribution of the $\mathrm{ZrO}_{2}-2$ particles when analysed by different analysis methods. These particles were non-spherical in morphology. Therefore the reported diameter values are those of the hydrodynamically equivalent sphere. Even though the diameter values are thus only the hydrodynamically equivalent diameter, their comparison in the light of different data analysis methods is still possible. Similar to the $\mathrm{ZrO}_{2}-1$ particles, the particle size distributions obtained by different analysis methods were very similar to each other. The onset as well as termination of the size distribution curves also matched well. Though the distribution from the GFL method was slightly broader than the other analysis methods, especially in the higher diameter range, the difference was still insignificant. A diameter value of $4.1 \mathrm{~nm}$ for the particles at the peak maximum was observed. It should also be noted that other possible techniques of size determination of the particles like dynamic light scattering, field-flow-fractionation or hydrodynamic chromatography were either not successful or were not suitable for particle characterisation owing to the small size of the zirconium dioxide particles, thus, leaving the analysis by analytical ultracentrifugation as the only accurate technique. The analysis with dynamic light scattering resulted in particle sizes from 6 to 40 nm owing to the negative impact of either dust or some aggregated particles on scattering. Particles in the range of $1000 \mathrm{~nm}$ were also observed for $\mathrm{ZrO}_{2}-1$ particles. These overall broad size distributions and higher particle sizes do not match with the microscopy findings. The resulting particle sizes from analytical ultracentrifugation were observed to match well with the microscopy findings, thus confirming 
the suitability of analytical ultracentrifugation for the analysis of particles with very small sizes. This also matches well with the recent findings on $\mathrm{ZnS}$ and $\mathrm{SiO}_{2} \cdot{ }^{[37,38]}$ It is remarkable that the differences in the particle size distributions of the samples analysed by various methods are small, although $\mathrm{dcd} t$ and $\left[l s-g^{*}(s)\right]$ did not apply diffusion correction in contrast to the other analysis methods.

Figure 4 demonstrates the particle size distributions of the $\mathrm{SiO}_{2}$ particles when analysed by different data analysis methods. The particles are observed to be more polydisperse in nature as compared to the zirconium dioxide particles. Also, in the case of the $\mathrm{SiO}_{2}$ particles, the different analysis methods exhibit more significant differences in the size distributions indicating greater extent of heterogeneity in these samples. The onset of the particle size distribution in the $\mathrm{SiO}_{2}-1$ particles (Figure 4a) was observed at considerably higher diameters in the $\left[l s-g^{*}(s)\right]$ analysis as
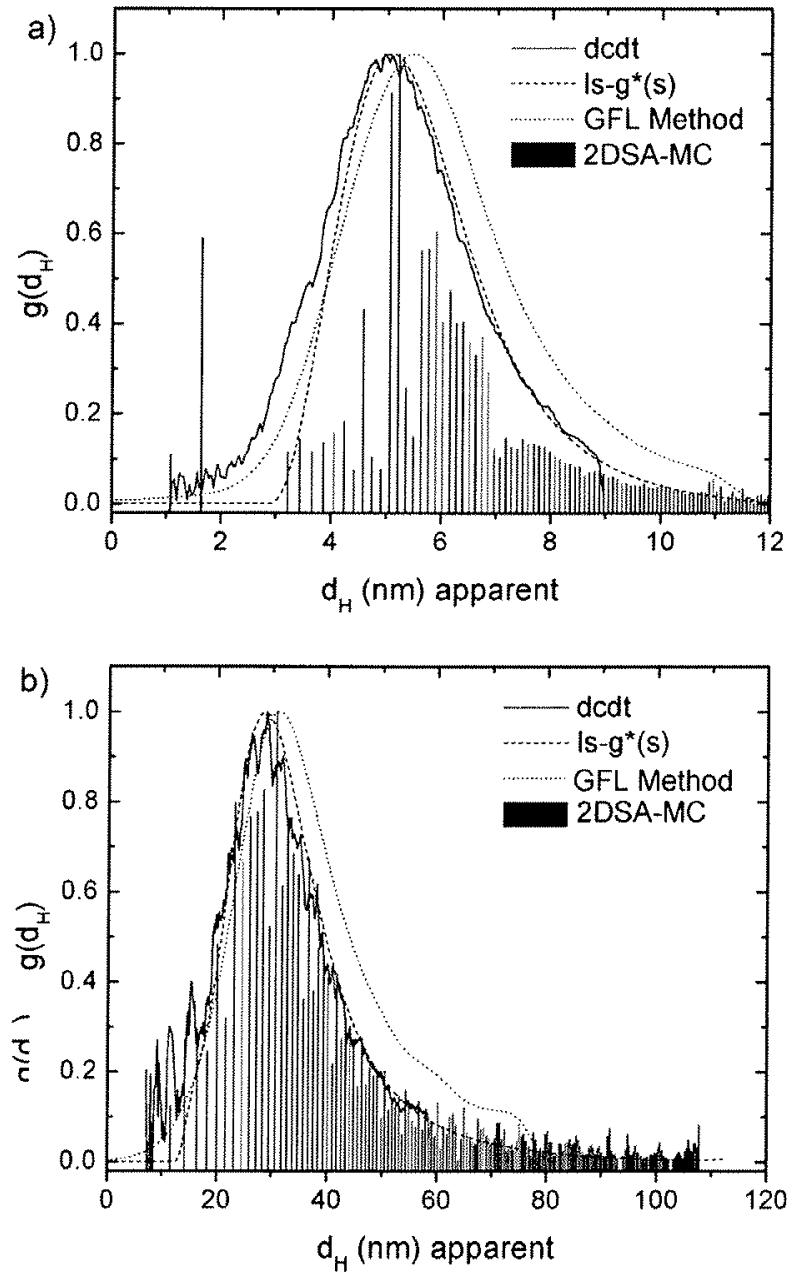

Figure 4. Particle size distributions of a) $\mathrm{SiO}_{2}-1$ and b) $\mathrm{SiO}_{2}-2$ particles using different sedimentation velocity data analysis programs. compared to the other methods. However, the peak diameters resulting from $\left[l s-g^{*}(s)\right], 2 \mathrm{DSA}-\mathrm{MC}$ and $\mathrm{d} c \mathrm{~d} t$ analysis were similar to be around $5 \mathrm{~nm}$. On the other hand the peak diameter and the size distribution were observed to shift in the case of the GFL method where a shift in the range of $0.4-0.5 \mathrm{~nm}$ in the peak particle size to higher sizes was observed. The shift of the size distributions in the GFI method was much more significant in the higher diameter range as the distribution was more or less in the same range as the other curves in the low diameter range. The distributions from other methods were similar to each other. The distribution in the case of dcdt analysis was however observed to abruptly terminate at a diameter of $9 \mathrm{~nm}$ owing to the requirement of the inclusion of the full scan range and both lower and higher plateaus from the integral scan in the analysis. There is also a possibility to use multispeed wide distribution analysis (WDA) in SedAnal for broader distributions. ${ }^{[39]}$ The $\mathrm{SiO}_{2}-2$ particles had the highest polydispersity of all investigated samples. The onset of the size distributions resulting from various analysis methods was very similar as shown in Figure $4 b$, though the $\left[l s-g^{*}(s)\right]$ distribution starts at higher diameter values. The distributions exhibited by $\left[1 s-g^{*}(s)\right]$, dcdt and 2DSA-MC were very similar, whereas the distribution from the GFL method was slightly shifted to the right as compared to the others. The peak maximum was shifted $3 \mathrm{~nm}(10 \%)$ to higher diameters in the distributions of 2DSAMC and the GFL method. Similar to Figure $4 a$, the shift of the GFL method was much more significant in the higher diameter range and the peak diameter was observed to shift by $3-4 \mathrm{~nm}$. The distribution from the GFL method was also broad as compared to $\left[l s-g^{*}(s)\right]$ and 2DSA-MC. The dcdt distribution was observed to be quite noisy in the smaller diameter range and it abruptly terminated at a diameter of $60 \mathrm{~nm}$ whereas the whole particle size range runs till roughly $110 \mathrm{~nm}$ owing to the above mentioned reasons. Thus, the inherent differences in the analysis methodologies result in slight differences in the particle size and size distributions when the analysed sample is polydisperse.

The morphology of the particles was confirmed by plotting a pseudo $3 \mathrm{~d}$ graph of the frictional ratio $f / f_{0}$ as a function of sedimentation coefficient in UltraScan. ${ }^{\text {[35] }}$ Figure 5 and 6 show these plots for $\mathrm{ZrO}_{2}$ and $\mathrm{SiO}_{2}$ particles, respectively. A majority of the $\mathrm{ZrO}_{2}-1$ particles, as shown in Figure $5 \mathrm{a}$, were observed to have a frictional ratio of unity thus confirming the spherical morphology of the particles. $\mathrm{ZrO}_{2}-2$ particles, on the other hand, have a significant fraction of particles with a frictional ratio of about 1.1, higher than unity thus confirming non-spherical morphology of the particles (Figure $5 b$ ). The $D$ and $s$ values inserted into the tool for prediction of axial ratios for four basic shapes in UltraScan yield an $a / b$ value of $\approx 3.4(a=5.4 \mathrm{~nm} /$ $b=1.6 \mathrm{~nm})$ for a prolate ellipsoid. ${ }^{[40,41]}$ Measuring a selection of particles on the TEM image (Figure $1 \mathrm{~b}$ ) give a 
a)

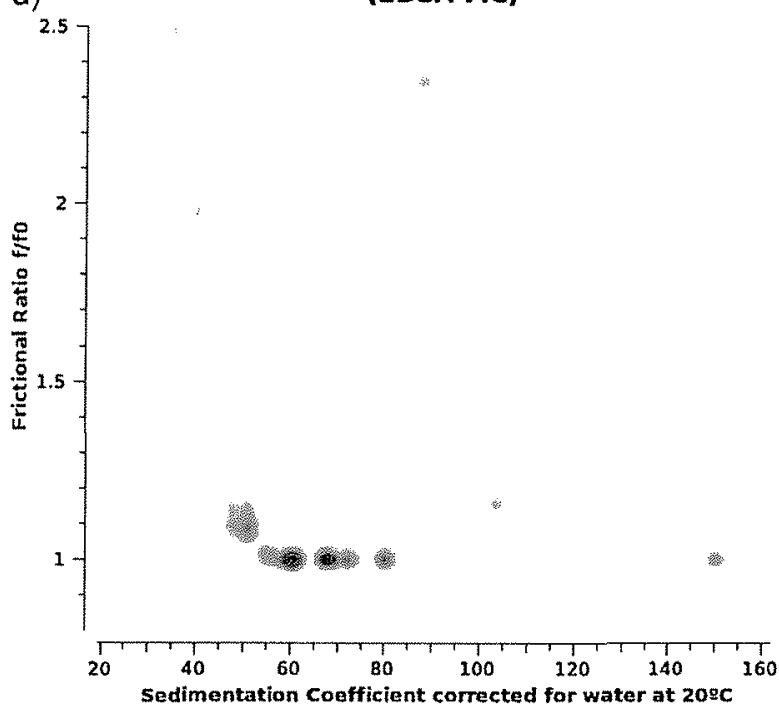

b)

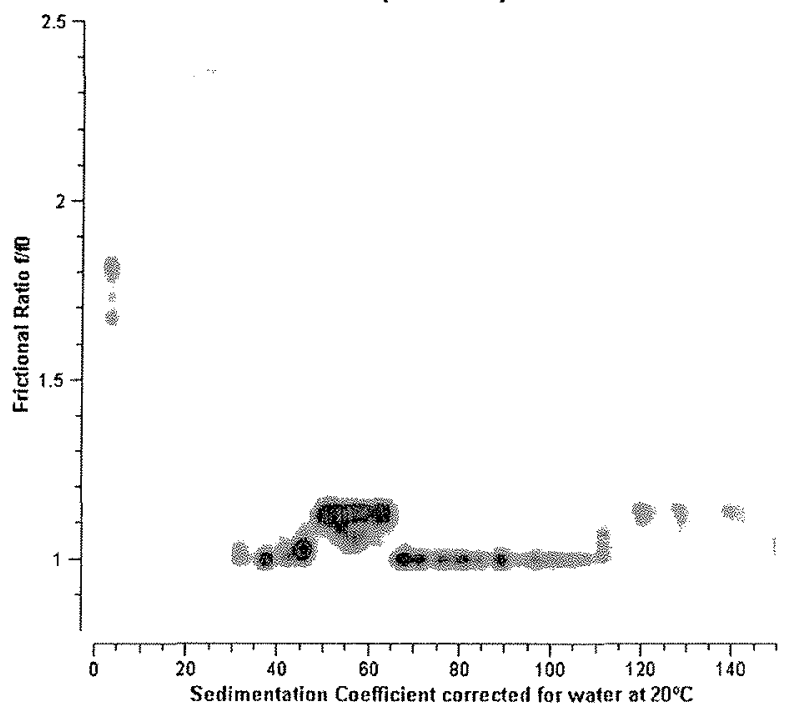

Figure 5. Pseudo 3d plots (UltraScan 2DSA-MC) showing the relation of frictional ratio with sedimentation coefficient for a) $\mathrm{ZrO}_{2-1}^{-1}$ and b) $\mathrm{ZrO}_{2}-2$ particles measured at a concentration of $0.5 \mathrm{mg} \cdot \mathrm{mL}^{-1}$.

ratio of $6.5 \mathrm{~nm} \times 2.4 \mathrm{~nm}$ for the prolates (square frames) and $6 \mathrm{~nm} \times 6 \mathrm{~nm}$ for the spherical particles (round frames) in reasonable agreement with the values from ultracentrifugation. Smaller particles cannot be seen in the image. In the case of $\mathrm{SiO}_{2}-1$ particles, a very small fraction of the sample had a frictional ratio in the range between 1.5 and 2.5 at low values of the sedimentation coefficient indicating nonspherical behaviour probably owing to the presence of some polymeric species (Figure 6a). The particles in the $\mathrm{SiO}_{2}-2$ sample, as shown in Figure $6 \mathrm{~b}$, had a frictional ratio of unity thus indicating the completely spherical morphol-

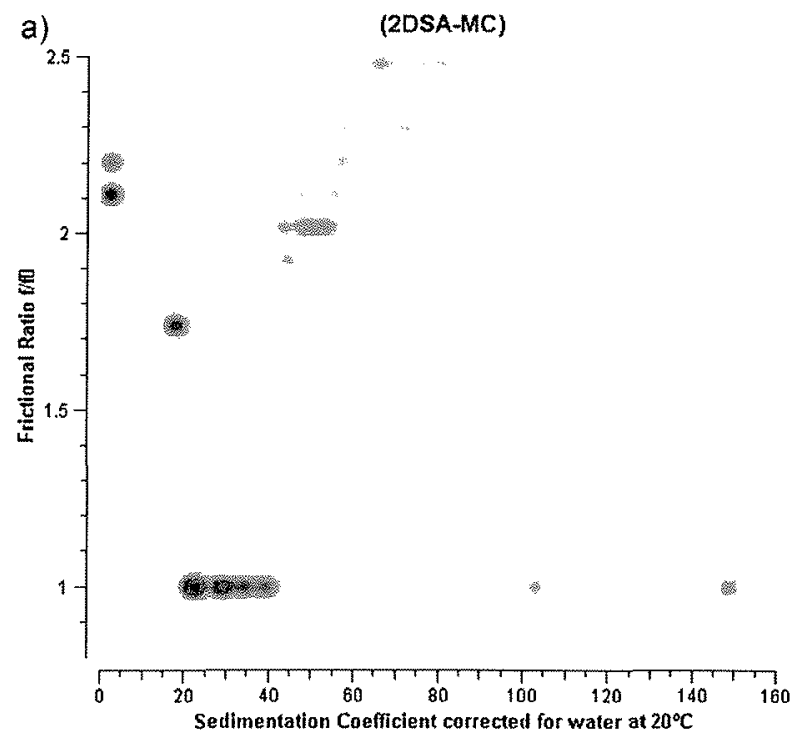

b)

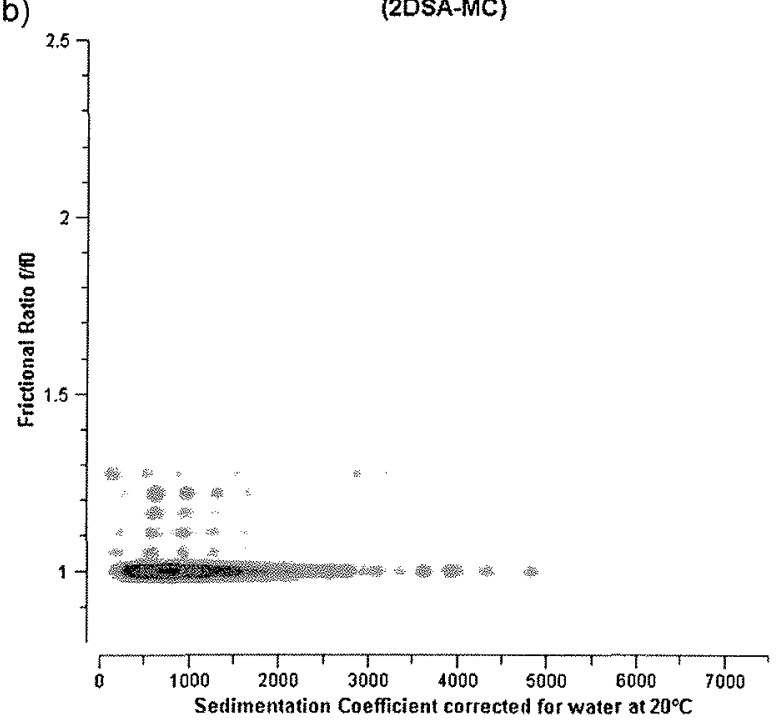

Figure 6. Pseudo 3d plots (UltraScan 2DSA-MC) showing the relation of frictional ratio with sedimentation coefficient for a) $\mathrm{SiO}_{2-1}$ and b) $\mathrm{SiO}_{2}-2$ particles measured at a concentration of $0.5 \mathrm{mg} / \mathrm{ml}$

ogy of the particles and absence of any other organic component dissolved or suspended in the aqueous phase. This also confirms that although the particles in the $\mathrm{SiO}_{2}-2$ sample were quite large and have a very broad size distribution, they were nonetheless spherical.

The concentration dependence of the particle size and its distribution was quantified by different analysis methods. $\mathrm{ZrO}_{2}-1$ particles were chosen for this purpose and were measured at concentrations of 2.5 and $5 \mathrm{~g} \cdot \mathrm{L}^{-1}$ in addition to the earlier used $0.5 \mathrm{~g} \cdot \mathrm{L}^{-1}$. Comparing the raw sedimentation data of the samples with higher concentration with 
those for the concentration of $0.5 \mathrm{~g} \cdot \mathrm{L}^{-1}$, it was observed that the data were much smoother at higher concentration and the number of fringes was also subsequently higher (Figure SI-II of Supporting Information). Thus, it is also of interest to determine if such improvement in the signal to noise ratio also results in differences in the size determination of the sample. Different concentrations did not lead to any significant changes in the particle size and its distribution thus indicating the concentration independence of the sedimentation data for spherical particles as expected (Figure SI-III of Supporting Information). All the different analysis methods allowed for the same conclusion and the data quality for the different concentrations was comparable. The frictional ratio of the particles measured at a concentration of $5 \mathrm{~g} \cdot \mathrm{L}^{-1}$ (Figure SI-IV of Supporting Information) was also observed to be unity similar to Figure $5 \mathrm{a}$ where the frictional ratio of the same particles measured at a concentration of $0.5 \mathrm{~g} \cdot \mathrm{L}^{-1}$ was plotted. This further indicates that the particle size and particle size distribution of the spherical nanoparticles had no concentration dependence.

The concentration independent sample $\mathrm{ZrO}_{2}-1$ sample (concentration $0.5 \mathrm{mg} \cdot \mathrm{mL}^{-1}$ ) was also used to compare the diffusion correction of the methods in SEDErT [ $c(s)]$, UltraScan (vHW and 2DSA-MC) and GFL (Figure 7). The incorporation of diffusion correction in the SEDFrT $c(s)$ analysis as well as the UltraScan vHW method were observed to narrow the distributions significantly compared to the GFL and 2DSAMC methods. Also in the vHW analysis parts of the upper and lower plateau are left out. The peak particle sizes differ between $4.7 \mathrm{~nm}$ (vHW), $4.5 \mathrm{~nm}(2 \mathrm{DSA}-\mathrm{MC})$ and $4.9 \mathrm{~nm}[\mathrm{c}(\mathrm{s})]$ and (GFL) which is a range of $9 \%$.

In summary, for the zirconium dioxide particles, which are comparatively more monodisperse in nature than the $\mathrm{SiO}_{2}$ particles, different analysis methods led to very similar

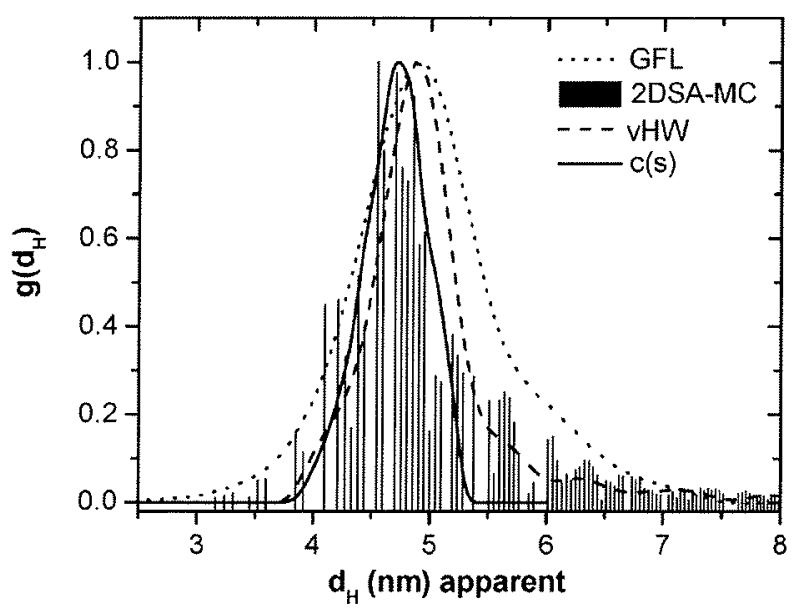

Figure 7. Particle size distributions of $\mathrm{ZrO}_{2}^{-1}\left(0.5 \mathrm{mg} \cdot \mathrm{mL}^{-1}\right)$ using different sedimentation velocity data analysis methods in order to compare the diffusion corrections. particle size distributions and the peak diameter also matched very well. The analysis of the more polydisperse silica particles exhibited more significant differences in the particle size distributions among different analysis methods. For the smaller sized $\mathrm{SiO}_{2}$ particles, the distributions from the GFL method were observed to shift by roughly $0.5 \mathrm{~nm}$ to the higher diameter range. For bigger particles, the distributions from the GFL method were broader as compared to $l s-g^{*}(s)$, dcdt and 2DSA-MC methods and also shifted to higher diameter range (by 10\%). The distributions from dcdt were observed to be noisy and abruptly ended without covering the whole range of the particle size.

\section{Conclusion}

All of the tested evaluation approaches and methods proved to be useable for the determination of particle size distributions but have different advantages and limitations. While SeDFrT can very effectively correct time and radius invariant noise with the possibility to effectively smooth the distributions, it can overcorrect the diffusion broadening of the sedimentation coefficient distribution $c(s)$ and assumes a constant $\bar{v}$. This can be a problem if samples with two different $\bar{v}$ are analysed. This is a common problem for the analysis of hybrid nanoparticles, which may not only have several components with different $\bar{v}$, but even a $\bar{v}$ distribution folded with a particle size distribution as demonstrated for the example of the iron storage protein ferritin. ${ }^{[42]}$ The van Holde-Weischet method implemented into UltraScan for the determination of the sedimentation coefficient distribution yields rather noisy distributions despite smoothing but has the advantage to be model free and requires no knowledge of other parameters like $f / f_{0}$ or $\bar{v}$. Ultrascan additionally allows for the simultaneous analysis of size and shape information, a feature which is also available in SEDFIT \{continuous $\left.\left[c\left(s, f_{0}\right)\right]\right\}$. The GFL method in VelXLAI is also a model free method and efficiently and rapidly predicts the size distributions of the relatively monodisperse particles. In the case of polydisperse particles, the distributions can be broader than the distributions observed from other methods. The time derivative method incorporated in SedAnal similarly generates more noise in the distribution when the particles are polydisperse in nature. In the case of monodisperse particles, the distributions are very smooth and virtually indistinguishable from the distributions predicted by other methods. The 2DSA-MC method also allows particle shape analysis and distributions also agree well with those from other methods.

Apart from the comparison of the different evaluation methods, the raw sedimentation data as well as the sedimentation coefficient distributions of the particles also provided useful information on the polydispersity of the particles. If a new evaluation method is introduced, the 
analyst should start with a standard which is well characterised. It has to be noticed, that the choice of scans, data range, smoothing and initial parameters has to be tested through overlaying of every evaluation. That way the influence of the modifications on the distribution can be revealed.

The subjective part of the user has to be mentioned also related to the shape of the distribution. Single species in a distribution can be identified by dilution. One concentration is not meaningful for a decision about real and artificial peaks. In summary the user should consider the values given in the different methods that indicate how the evaluated distribution fits the raw dataset (rmsd, residuals map, overlay of the raw data with the model, statistics).

The results of this method comparison should be transferable to all nanoparticle systems including drug nanoparticles, biopolymer hybrid particles, biologically active nanoparticles and so forth. In addition to the already established application in biophysical chemistry of biopolymers and their interactions, this will now enable the efficient use of Analytical Ultracentrifugation in a large variety of research areas involving nanoparticles including the large field of Biosciences.

Acknowledgements: The authors are thankful to M. Page at BASF for excellent experimental support and Rona Pitschke and Heike Runge at the MPI KGF for the TEM pictures. The Max Planck Society is acknowledged for financial support. We also want to thank the programmers of the software packages that were used: UltraScan calculations were performed on the UltraScan LIMS cluster at the Bioinformatics Core Facility at the University of Texas Health Science Center at San Antonio and the Lonestar cluster at the Texas Advanced Computing Center supported by NSF Teragrid Grant \#MCB070038 (to Borries Demeler). B. Demeler, M. D. Lechner, P. Schuck and W. F. Stafford and their co-workers for the support and the ongoing development. All of these programs except VelXLAI are available for free via the internet.

Keywords: analytical ultracentrifugation; gosting fujita lechner method; lamm Equation solutions; nanoparticles; particle size distribution; time derivative method; two dimensional spectrum analysis; van Holde-Weischet

[1] T. Svedberg, J. B. Nichols, J. Am. Chem. Soc. 1923, 45, 2910.

[2] T. Svedberg, H. Rinde, J. Am. Chem. Soc. 1924, 46, 2677.

[3] W. Mächtle, L. Börger, Analytical Ultracentrifugation of Polymers and Nanoparticles, Springer, Berlin 2006.

[4] H. Cölfen, "Analytical ultracentrifugation of colloids", in: Analytical Ultracentrifugation: Techniques and Methods, D. J. Scott, S. E. Harding, A. J. Rowe, Eds., The Royal Society of Chemistry, Cambridge 2005, p. 501.

[5] T. M. Laue, Prog. Colloid Polym. Sci. 1994, 94, 74.

[6] H. K. Schachman, Ultracentrifugation in Biochemistry, Academic Press, New York 1959.
[7] R. Giebeler, "The Optima XL-A: A New Analytical Ultracen trifuge with a Novel Precision Absorption Optical System", in: Analytical Ultracentrifugation in Biochemistry and Polymer Science, S. E. Harding, A. J. Rowe, J. C. Horton, Eds., The Royal Society of Chemistry, Cambridge 1992, p. 16-25.

[8] S. Hanlon, K. Lamers, G. Lauterbach, R. Johnson, H. K. Schachman, Arch. Biochem. Biophys. 1962, 99, 157.

[9] R. L. Baldwin, J. W. Williams, J. Am. Chem. Soc. 1950, 72, 4325.

[10] H. Fujita, Mathematical Theory of Sedimentation Analysis, Academic Press, New York 1962

[11] M. D. Lechner, W. Mächtle, Makromol Chem., Makromol. Symp. 1992, 61, 165.

[12] T. Svedberg, K. O. Pedersen, The Ultracentrifuge, Oxford University Press, London 1940.

[13] W. Mächtle, Biophys. J. 1999, 76, 1080

[14] L. A. Holladay, Biophys. Chem. 1979, 10, 187.

[15] J. Behlke, O. Ristau, Progr. Colloid Polym. Sci. 1997, 107, 27.

[16] J. Behlke, O. Ristau, Biophys. J. 1997, 72, 428.

[17] J. Philo, Biophys. J. 1997, 72, 435.

[18] P. Schuck, D. B. Millar, Anal. Biochem. 1998, 259, 48.

[19] P. Schuck, Biophys. J. 2000, 78, 1606.

[20] P. Schuck, M. A. Perugini, N. R. Gonzales, G. J. Howlett, D. Schubert, Biophys. J. 2002, 82, 1096.

[21] E. Brookes, W. Cao, B. Demeler, Eur. Biophys. J. 2010, 39, 405.

[22] P. H. Brown, P. Schuck, Biophys I. 2006, 90(12), 4651.

[23] K. E. van Holde, W. O. Weischet, Biopolymers 1978, 17, 1387.

[24] B. Demeler, H. Saber, J. C. Hansen, Biophys. J. 1997, 72, 397.

[25] J. Geiselmann, T. D. Yager, S. C. Gill, P. Camettes, P. H. von Hippel, Biochemistry 1992, 31, 111.

[26] S. C. Gill, T. D. Yager, P. H. von Hippel, J. Mol. Biol. 1991, 220, 325.

[27] H. Fujita, Foundations of Ultracentrifugal Analysis, Wiley, New York 1975.

[28] L. J. Gosting, J. Am. Chem. Soc. 1952, 74, 1548.

[29] J. H. Kehrhahn, M. D. Lechner, W. Mächtle, Polymer 1993, 34, 2447.

[30] M. D. Lechner, W. Mächtle, Progr. Colloid Polym. Sci. 1999, 113, 37.

[31] W. F. Stafford, "Methods for obtaining sedimentation coefficient distributions", in: Analytical Ultracentrifugation in Biochemistry and Polymer Science, S. E. Harding, A. J. Rowe, I. C. Horton, Eds., The Royal Society of Chemistry, Cambridge 1992, p. 359.

[32] D. A. Yphantis, Biophys. J. 1984, 45, 324.

[33] W. F. Stafford, Anal. Biochem. 1992, 203. 295.

[34] G. Garnweitner, Zirconia Nanomaterials: Synthesis and Biomedical Application, in: Nanostructured Oxides Vol. 2, C. Kumar, Ed., Wiley-VCH, Weinheim, Germany 2009.

[35] G. Garnweitner, L. M. Goldenberg, O. V. Sakhno, M. Antonietti, M. Niederberger, J. Stumpe, Small 2007, 3, 1626.

[36] CRC Handbook of Chemistry and Physics, $73^{\text {rd }}$ edition, D. Lide, Ed., CRC Press, Cleveland, Ohio 1992.

[37] Y. Dieckmann, H. Cölfen, H. Hofmann, A. Petri-Fink, Anal. Chem. 2009, 81, 3889.

[38] K. L. Planken, B. W. M. Kuipers, A. P. Philipse, Anal Chem. $2008,80,8871$

[39] W. F. Stafford, E. H. Braswell, Biophys. Chem. 2004, 108, 273.

[40] K. E. van Holde, W. C. Johnson, P. S. Ho, Principles of Physical Biochemistry, $2^{\text {nd }}$ edition, Intl. ed., Prentice-Hall, Englewood Cliffs, NJ 2005.

[41] C. R. Cantor, P. R. Schimmel, Biophysical Chemistry, Freeman Part II, San Francisco, CA 1980

[42] A. Völkel, H. Cölfen, Eur. Biophys. J. 2003, 32, 432. 